\title{
Considerations Underlying Parents' Acceptance of COVID-19 Vaccines for Their Child: Evidence from Denmark
}

\author{
Frederik Juhl Jørgensen* \& Michael Bang Petersen \\ Department of Political Science, Aarhus University \\ *Corresponding author: fj@ps.au.dk
}

\begin{abstract}
Background: Since the initial phases of vaccination campaigns, health authorities across the world have recommended vaccination of children between 15 and 18; and since the summer of 2021, vaccinations of children between 12 and 15 have been recommended. Recently, American and European Health Authorities have approved the use of a vaccine against COVID-19 for children between age 5 and 11 . In this article, we ask what predicts parents' intention to vaccinate their children against COVID-19.

Methods: We use empirical data from a large-scale survey in Denmark about vaccine intentions. The survey included a subsample of 794 parents to children aged 6-15. For this group of parents, we assess key predictors (including demographics, predispositions, vaccine perceptions, and vaccine barriers) of parents' acceptance of a vaccine for their child against COVID-19.
\end{abstract}

\section{Results:}

We propose that the vaccine decision follows a stepwise process: demographic variables shape parents' predispositions, which again shape their specific perceptions of the safety and effectiveness - both in general and for their children - of the particular vaccine. Moreover, we argue that vaccine barriers will moderate this process. Our results demonstrate that parents' intention to get their children vaccinated against COVID-19 is not driven by considerations regarding the disease of COVID-19 but by considerations of the safety of vaccines and added benefits of vaccination such as normalizing societal and everyday life. The balance of these considerations shift depending on the child's age, with lower acceptance for younger children. Furthermore, the content of considerations are shaped by trust in the health authorities and factual vaccine knowledge.

Conclusion: To increase parents' acceptance of a COVID-19 vaccine for their children, health authorities should focus on establishing and communicating evidence for the safety of vaccines and the impact of vaccination for their everyday lives.

Keywords: COVID child vaccination, public health, survey research 


\section{Key Messages}

\section{Implications for policy makers}

- Parents' acceptance of a COVID-19 vaccine falls markedly among younger children

- Communication to increase vaccine acceptance among parents for their children should focus on removing safety concerns and establish an understanding how the vaccine will benefit the child in terms of normalizing everyday life

- Communication about the severity of the disease may not strongly influence vaccine acceptance, potentially given the generally lower risk from COVID-19 among children

- Practical barriers including the child's fear of needles and disagreement among parents about the vaccination decision do not strongly impact vaccine acceptance, potentially because parents are used to taking complex decisions on behalf of their child

- These results were obtained in Denmark, a country with a high adult acceptance of COVID-19 vaccines, and, hence, hesitancy and concerns among parents may be larger in other countries

\section{Implications for public}

The findings show that many parents in Denmark, a country with generally high acceptance of COVID-19 vaccines, a torn between different considerations when deciding whether their child should be vaccinated against COVID-19. Parents are motivated to vaccinate their children in order to normalize society and the child's own everyday life but are hesitant due to concerns about side-effects from the vaccines. The balance of these considerations favor vaccination among parents of older children but hesitancy increase among parents of younger children. The results indicate that it is not atypical for parents to have countervailing considerations about the decision to vaccinate one's child against COVID-19. 


\section{Background}

During the COVID-19 pandemic, parents and caretakers of children face decisions about whether or not to let their children be vaccinated against COVID-19. Health authorities across the world have been recommending vaccination of children between 15 and 18 since the initial phases of the vaccination campaigns and, since the summer of 2021, vaccinations of children between 12 and 15 have also been recommended across countries. Recently, American and European Health Authorities have approved the use of a vaccine against COVID-19 for children between age 5 and 11.

The pandemic has revealed substantial hesitancy towards COVID-19 vaccines in some countries. This hesitancy may be greater in the case of children who historically have not been at serious risk of severe illness from COVID-19. At the same time, the circulation of variants of the coronavirus with increased transmissibility implies that both the personal and the societal benefits may outweigh the costs of vaccination. Thus, with the emergence of the delta variation, the number of COVID-19 cases among children have been rising markedly (Tanne, 2021) Furthermore, it is now clear that for the society to reach herd immunity and hinder the spread of COVID-19 pandemic without any other non-pharmaceutical measures, it is required that a substantial proportion of the entire population achieves immunity either through vaccination or natural infection (Britton et al., 2020; Bartsch et al., 2020; Sanche et al., 2020). Accordingly, it is of pressing importance to understand the exact considerations underlying parents' decisions to vaccinate their children against COVID-19, in order to facilitate effective and empowering health communication.

Some early studies have already investigated parents' intention to vaccinate their children. These studies point to several background characteristics that are associated with parents' intention to vaccinate their children against COVID-19. Increased vaccine willingness is demonstrated to be associated with older age of children (Goldman et al., 2020; Szilagyi et al., 2021) as well as their parents (Kelly et al., 2021; Montalti et al., 2021; Skjefte et al., 2021). Similarly, intended vaccine uptake increase when fathers complete the questionnaire (Goldman et al., 2020; Kelly et al., 2021; Montalti et al., 2021; Scherer et al., 2021; Teasdale et al., 2021; Yigit et al., 2021). Higher socioeconomic status is related with an increase in COVID-19 vaccine willingness. Specifically, higher educated parents (Brandstetter et al., 2021; Hetherington et al., 2021; Kelly et al., 2021; Montalti et al., 2021; Scherer et al., 2021; Skjefte et al., 2021; Szilagyi et al., 2021; Teasdale et al., 2021) and higher income parents (Hetherington et al., 2021; Kelly et al., 2021; Skjefte et al., 2021; Teasdale et al., 2021) are more likely to accept vaccination of their child compared to low educated and income parents, respectively. Moreover, some studies observe ethnic differentials in 
vaccine intentions with minority groups being more hesitant to vaccinate their children against COVID-19 (S. Bell et al., 2020; Kelly et al., 2021; Scherer et al., 2021).

Studies also show that parents who have more confidence in public health authorities and health research are more likely to accept vaccination of their children (Brandstetter et al., 2021; Skjefte et al., 2021). Similarly, parents' knowledge about COVID-19 protective measures, compliance with authorities' recommendations, and general level of information about the pandemic are positively associated with their intention to vaccinate their children (Brandstetter et al., 2021; Skjefte et al., 2021).

Positive perceptions of vaccines predict parents' willingness to vaccinate their children against COVID-19 positively. Aligned with research on adults own willingness to take up COVID19 vaccines (Kaplan, R. M., \& Milstein, 2021; Schwarzinger et al., 2021; Motta et al., 2020; Kreps et al. 2020; Jørgensen et al., 2021), general confidence in vaccines, and more specifically confidence in the efficacy and safety of vaccines against COVID-19 as well as parent COVID-19 vaccine uptake positively predict parents' intention to vaccinate their children (Goldman et al., 2020; Hetherington et al., 2021; Kelly et al., 2021; Ruggiero et al., 2021; Skjefte et al., 2021; Szilagyi et al., 2021; Yilmaz \& Sahin, 2021; Zhang et al., 2020). In contrast, concerns over side effects and lack of efficacy are associated with a decrease in parents' willingness to vaccinate their children (Montalti et al., 2021; Ruggiero et al., 2021; Zhang et al., 2020). Similarly, parents' that are generally more worried over COVID-19 are more likely to willingly accept vaccination of their children (Kelly et al., 2021; Skjefte et al., 2021; Yigit et al., 2021; Yilmaz \& Sahin, 2021).

We contribute in several ways to the existing studies. First, while the existing studies point to key predictors of child vaccine willingness, most of the studies only observed a subset of the relevant predictor. Consequently, the combined findings simultaneously show mixed evidence for the individual predictors. We collect information of a wide range of predictors - that have been proposed in these prior studies - that we are able to integrate into one model. Second, as we discuss below, we propose (and test) a theoretical model of how the different predictors should be factored in when trying to explain child vaccine willingness. More concretely, we propose a specific sequencing of the relevant predictors for explaining parents' COVID-19 vaccine willingness of their children. Third, the overwhelming majority of previous studies are based on convenience sampling (Galanis et al., 2021). As is well known this potentially renders the conclusions from these studies vulnerable to selection biases when trying to make inference to the underlying populations. To the contrary, our data is based on a probability sample drawn from the entire database of Danish social security 
numbers and supplied by Statistics Denmark (DST). Fourth, our focus on Denmark supplies a context with comparatively very high vaccine uptake in the adult population but, even here, reveals substantial hesitancy towards especially younger children This suggests that this may present an important barrier for in other contexts too.

Figure 1. Theoretical model of vaccine willingness

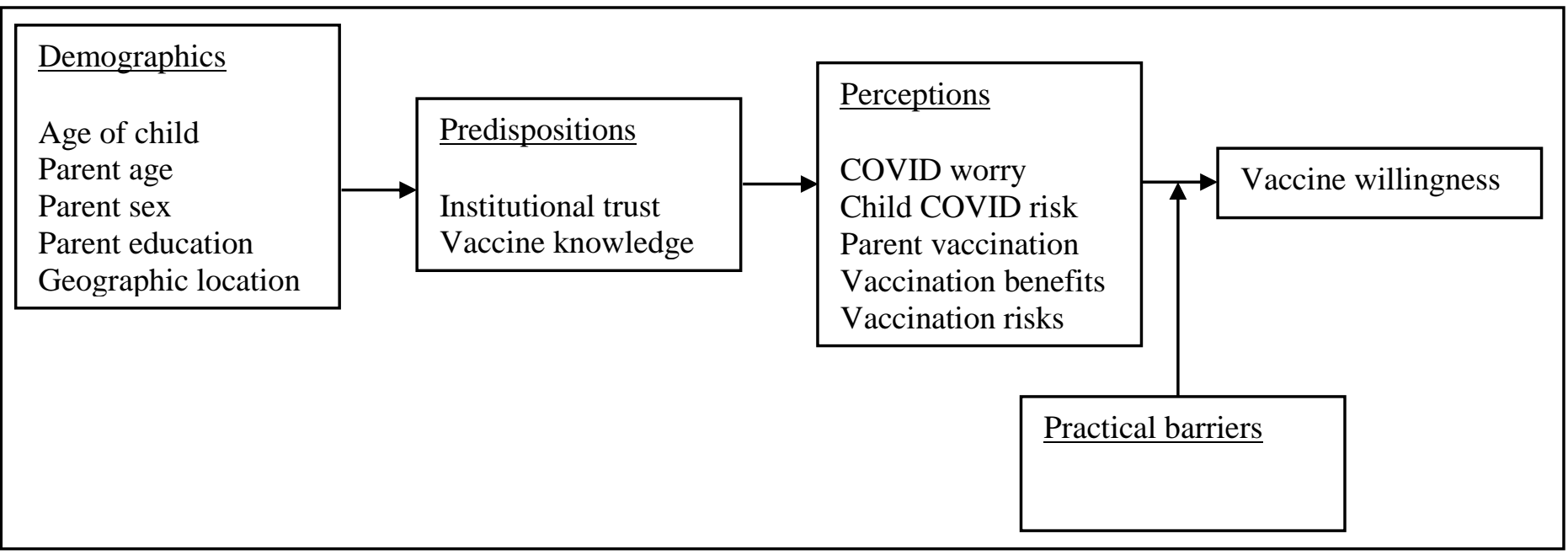

The above review of the key factors underlying vaccination decisions among parents are in line with the major theoretical frameworks for understanding vaccine acceptance more generally. These frameworks converge on arguments that (a) trust in the approving institutions are required to inspire confidence in the vaccine's effectiveness and safety; (b) that people need to be sufficiently concerned about the disease to remove complacency and, hence, make it a worthwhile calculation to receive vaccination; and (c) that the resulting intention to become vaccinated might be affected by matters of convenience in the form of practical barriers that disables an individual from acting on their intention to get vaccinated (WHO, 2014; see also Betsch et al., 2018).

In the theoretical literature, these factors are often analyzed as factors that enter the vaccination decision-making process concomitantly (e.g., Betsch et al., 2018). Yet, as is clear from the larger literature on the psychology of attitudes, it is helpful to view decision-making as a stepwise process where some earlier considerations color other later considerations (Campbell et al., 1980; Zaller, 1992). For example, demographic variables such as the gender and age of parents will shape their psychological predispositions such as their general confidence in the health authorities, which again will shape their specific perceptions of the safety and effectiveness - both in general and for their children - of this particular vaccine approved by these authorities. 
In the specific context of vaccination intentions, this stepwise process is further complicated by the role of practical barriers to convenience. Thus, even parents who perceive vaccines to be safe and effective, may face more practical obstacles. In the literature on vaccine hesitancy, there are often a focus on matters such as competing demands on time and location and opening hours of vaccination centers. In this case of children, however, other barriers may hinder a parent from acting on their vaccination intention such as conflicting views from the other parent or concerns among the children themselves (e.g., fear of needles). Thus, vaccination decisions among children are special in that they often require a coordinated agreement among the three parties of two parents and a child. Analytically, such barriers may best be thought of as moderating the influence of vaccine perceptions on intentions. Among those with fewer barriers, there should be a greater alignment between perceptions and intentions. Among those facing significant practical barriers, however, the association between perceptions and intentions may be substantially decreased.

Figure 1 demonstrates the stepwise logic of the proposed analysis in order to understand the decision-making process underlying parents' decision to vaccinate their children against COVID-19. Figure 1 also displays the particular variables that will be analyzed at each step. The content of these variables will be described below.

\section{Methods}

\section{Data}

Our data is collected by the survey firm Epinion for the Danish Health Authority. The survey was designed in collaboration between Epinion, the Danish Health Authority and one of the study authors. Epinion provided initial analyses of these data for the Danish Health Authority. In this manuscript, we extend those initial analyses.

Participants were recruited using simple random sampling among the Danish public aged 18 or older based on the entire database of Danish social security numbers, supplied by Statistics Denmark (DST). The recruited participants can thus be viewed as representative of the broader Danish population. On August 31, 2021 24,961 individuals were invited to take the survey. These randomly selected individuals received a letter that invited them to participate in the study via eBoks (a nation-wide Danish electronic mail system). If they had not taken the survey within four days, they received a letter reminding them about the study also via eBoks (on September 4). No monetary incentives were employed. Altogether, 6,879 individuals participated in the study between August 31 and September 8 , yielding a response rate of about 28 percent. Note that about 8 percent, mainly older 
people, of the Danish population are exempt from eBoks. In this study, we focus on parents with children aged 6 to 15 years old who answered questions about their willingness to vaccinate their child $(\mathrm{N}=791)$. Table 1 shows the sample characteristics.

\section{Measures}

Prior to answering questions related to their willingness to vaccinate their children, respondents were filtered such that only the relevant group of parents (with children in the ages 6 to 15 years) were asked to fill in this part of the questionnaire. Moreover, if parents had more than one child within the age groups 6-15 years we particularly asked respondents to answer the questions about vaccine willingness based on the child who most recently had a birthday. Altogether, this means that the estimates in, for example, Figure 2 below cannot be interpreted as the share of children who are willing to vaccinate. Instead, the estimates denote the share of parents whose children are vaccinated or plan to become vaccinated if it is recommended to them.

\section{Outcome}

Our outcome, parents' willingness to vaccinate their child against COVID-19, is constructed by combining two questions. First, we ask parents if their child has been vaccinated against COVID-19. Parents had three response options: (1) "Yes, the child has received the first shot", (2) "Yes, the child has received the first and second shot", and (3) "No". If parents responded no, they were guided to the second question about willingness which was about whether they plan to get their child vaccinated or not (for children below the age of 12, this question was framed in the context of a vaccine that was recommended by the Danish Health Authority). Again, parents had three response options: (1) "Yes, I plan for the child to be vaccinated", (2) "No, I have doubts whether the child should be vaccinated or not, and (3) "No, I am certain that the child should not be vaccinated". Responses are categorized as vaccine acceptance (and coded as 1) if the parent response was (1) or (2) on the first question or (1) on the second. Otherwise, responses are coded as 0.

\section{Predictors of vaccine willingness}

We categorize our set of predictors into four groups, including demographics, predispositions (institutional trust and knowledge), vaccine perceptions, and barriers for vaccination.

The demographic variables include age of the child, the sex of the parent, the age of the parent, the education of the parent, and the geographic region. Age of the child is split into three categories: 
the young (6-9 years), the medium-aged (10-12 years), and the older children for whom the vaccine was approved by the time of the survey (13-15 years). Parents are grouped into three age categories: young (18-34 years), medium-aged (35-50 years), and older parents (51-65 years). Parents are similarly classified as either low educated (primary school and vocational training) or high educated (high school and higher education). Geographic region, is the five regions of Denmark (the Municipality, Sjælland, Syddanmark, Midtjylland, and Nordjylland).

On predispositions, we focus on trust in the relevant health institutions and knowledge about the vaccines. More specifically, respondents were asked to "Give your assessment on a scale from 0 to 10 , where 0 means that you have no trust at all, and 10 means that you have full trust". Then, they assessed their trust in the "Danish Health Authority" and "research in health and pharmaceuticals", respectively. We add the responses on these two questions together to form a scale of institutional trust $(r=.73)$. Knowledge is captured by eight factual statements about the coronavirus vaccines. For example, "it has been proven that the vaccines can affect your fertility". We recode responses on each of the statements as correct (coded 1) or incorrect (coded 0) and obtain a score for each participants' knowledge by adding together responses on all the statements.

On vaccine perceptions, we focus on five perceptions, including parent vaccination against COVID-19, general COVID worry, child COVID worry, beliefs about the benefits of child vaccination, and beliefs about the risks of child vaccination. Parent vaccination is based on a question that asks: "Are you vaccinated against COVID-19"? Responses are coded 1 if the parent said "yes" and 0 if they said "no" (note that 96 percent of the parents in our sample indicated that they were vaccinated themselves). For general COVID worry, we form a scale by adding together a question that ask parents whether they are very worried about getting infected with COVID-19 and a question that ask whether they are very worried that COVID-19 the next couple of years will be a threat to the Danish society $(\mathrm{r}=.57)$. For child COVID worry, we form a scale $(\alpha=.61)$ by adding together respondents' agreement with three statements: (1) I am worried that my child will be infected with COVID-19, (2) Children only very rarely get serious sick from COVID-19, and (3) Children only very rarely experience late consequences from being sick with COVID-19. For child vaccination benefits, we form a scale $(\alpha=.67)$ by adding together parents agreement with three statements: (1) Being vaccinated against COVID-19 will create a more normal everyday life for my child, (2) The vaccine against COVID-19 in the Danish vaccine program provides good protection against COVID19 among children, and (3) The most important for me is that my family contributes to the protection of others in the society against COVID-19. For child vaccination worries, we form a scale $(\alpha=.70)$ 
by adding together parents agreement with six statements: (1) Vaccines against COVID-19 can lead to developmental disorders among children, (2) Vaccines against COVID-19 can have unknown longterm side-effects among children, (2) I have heard about many children that suffer from severe sideeffects of a COVID-19 vaccine, (4) In Denmark we should wait and see if you discover new sideeffect from COVID-19 vaccines before we vaccinate children, (5) If my child is vaccine against COVID-19, then it will likely require that the child is vaccinated every year in the future, and (6) When it comes to children, then immunity from infection with COVID-19 is to prefer over vaccine immunity. For all questions on perceptions, respondents stated their agreement on scales from 1 (completely disagree) to 7 (completely agree). They also had the option of responding, "Don't know". For each of the items, we recode a "Don't know" response into the neutral category ("Neither agree nor disagree"). Note that the estimates are robust to list wise deletion of "Don't know" responses (see Figure S.2).

For barriers of vaccination, we focus specifically on practical barriers and form a barrier scale ( $\alpha=.43$ ) from respondents' agreement across five statements: (1) It is difficult to find time for vaccination of children in a stressful everyday life, (2) My child is afraid of needles, (3) My child is afraid of side-effects from the vaccine against COVID-19, (4) The majority of my child's friends are vaccinated against COVID-19, and (5) I disagree with my child's other parent about vaccination of our child. For all barrier statements, respondents stated their agreement on scales from 1 (completely disagree) to 7 (completely agree) and also had a "Don't know" response option. For each of the items, we recode a "Don't know" response into the neutral category ("Neither agree nor disagree"). Note that the estimates are robust to list wise deletion of "Don't know" responses (see Figure S.2).

We rescale all the continuous predictors with mean 0 and a standard deviation of .5 . In the results below, this implies that the coefficients on the continuous predictors can be interpreted as the change in the likelihood of accepting the vaccine associated with a 2 standard deviations change in the predictors. Table 1 shows the descriptive statistics for all the predictors above.

\section{Statistical modelling}

To analyze the relationship between parents' willingness to vaccinate their children and the predictors, we utilize OLS regression models that regress vaccine willingness on the proposed predictors. More specifically, we provide results from multivariate regression models that sequence the predictors according to the theoretical model proposed above. In the first model, we regress willingness on our battery of demographics; in the second model, we factor in predispositions (trust 
and knowledge); in the third mode, we introduce perceptions (general covid worry, child covid risk, parent vaccination, child benefits from vaccination, and child costs from vaccination); in the fourth model, we include vaccine barriers as the final predictor; in the fifth model, we rerun the model with all predictors while splitting our sample into parents' below and above the median with respect to perceived barriers to vaccination of the child. In the main results, we report estimates from OLS regression models. Note, however, that we re-estimate all findings using logistic regression. In the SI, we report the estimated logistic regression coefficients. All findings replicate (see Figure S.1).

Table 1. Descriptive statistics

\begin{tabular}{lcccc}
\hline & Mean & SD & Min & Max \\
\hline Child age & & & & \\
6-9 years & 0.27 & 0.45 & 0.00 & 1.00 \\
10-12 years & 0.32 & 0.47 & 0.00 & 1.00 \\
13-15 years & 0.41 & 0.49 & 0.00 & 1.00 \\
Demographics & & & & \\
Sex: male & 0.41 & 0.49 & 0.00 & 1.00 \\
Education: high & 0.75 & 0.43 & 0.00 & 1.00 \\
Age: 18-34 & 0.05 & 0.22 & 0.00 & 1.00 \\
Age: 35-50 years & 0.80 & 0.40 & 0.00 & 1.00 \\
Age: 51-65 years & 0.15 & 0.36 & 0.00 & 1.00 \\
Municipality & 0.25 & 0.43 & 0.00 & 1.00 \\
Region: Nordjylland & 0.11 & 0.31 & 0.00 & 1.00 \\
Region: Sjælland & 0.14 & 0.35 & 0.00 & 1.00 \\
Region: Syddanmark & 0.19 & 0.39 & 0.00 & 1.00 \\
Perceptions & & & & \\
Parent vaccinated & 0.96 & 0.19 & 0.00 & 1.00 \\
Trust & -0.00 & 0.50 & -2.78 & 0.48 \\
Knowledge & -0.00 & 0.50 & -1.87 & 0.49 \\
General covid worry & -0.00 & 0.50 & -0.98 & 0.93 \\
Child covid worry & -0.00 & 0.50 & -0.99 & 1.48 \\
Vaccine benefits & -0.00 & 0.50 & -1.69 & 0.61 \\
Vaccine costs & 0.00 & 0.50 & -1.32 & 1.46 \\
Barriers & & & & \\
Vaccine barriers & 0.00 & 0.50 & -0.87 & 1.51 \\
\hline
\end{tabular}

\section{Results}

Overall 74.7 percent (95 \% CI: 71.7-77.7) of the parents' in our sample report a willingness to have their child vaccinated against COVID-19. In comparison, Galanis et al. (2021) - in a meta-analysisfind that previous studies in other contexts estimate an overall willingness of 56.8 percent (95\% CI: 51.8-61.8) of parents who intend to vaccinate their children against COVID-19. Estimates of vaccine 
willingness, however, varied substantially in the studies underlying this meta-analysis: more specifically, parents' willingness ranged from 29 percent (Yigit et al., 2021) to 72.7 percent (Xu et al., 2021). This shows that parents' child vaccine willingness - just like the overall vaccine uptakeis overall high in Denmark compared to many other contexts. ${ }^{1}$ Figure 2, however, shows that vaccine willingness varies substantially with the child's age. More specifically, in the 13-15 years age group 89.6 percent (95\% CI: 86.2-92.9) of the parents report that they are willing to vaccinate their child. This drops to 71.7 (95 \% CI: 66.1-77.3) and 56.2 (95\% CI: 49.6-62.9) percent in the 10-12 and 6-9 years age groups, respectively.

Figure 2. Child vaccine willingness, by age

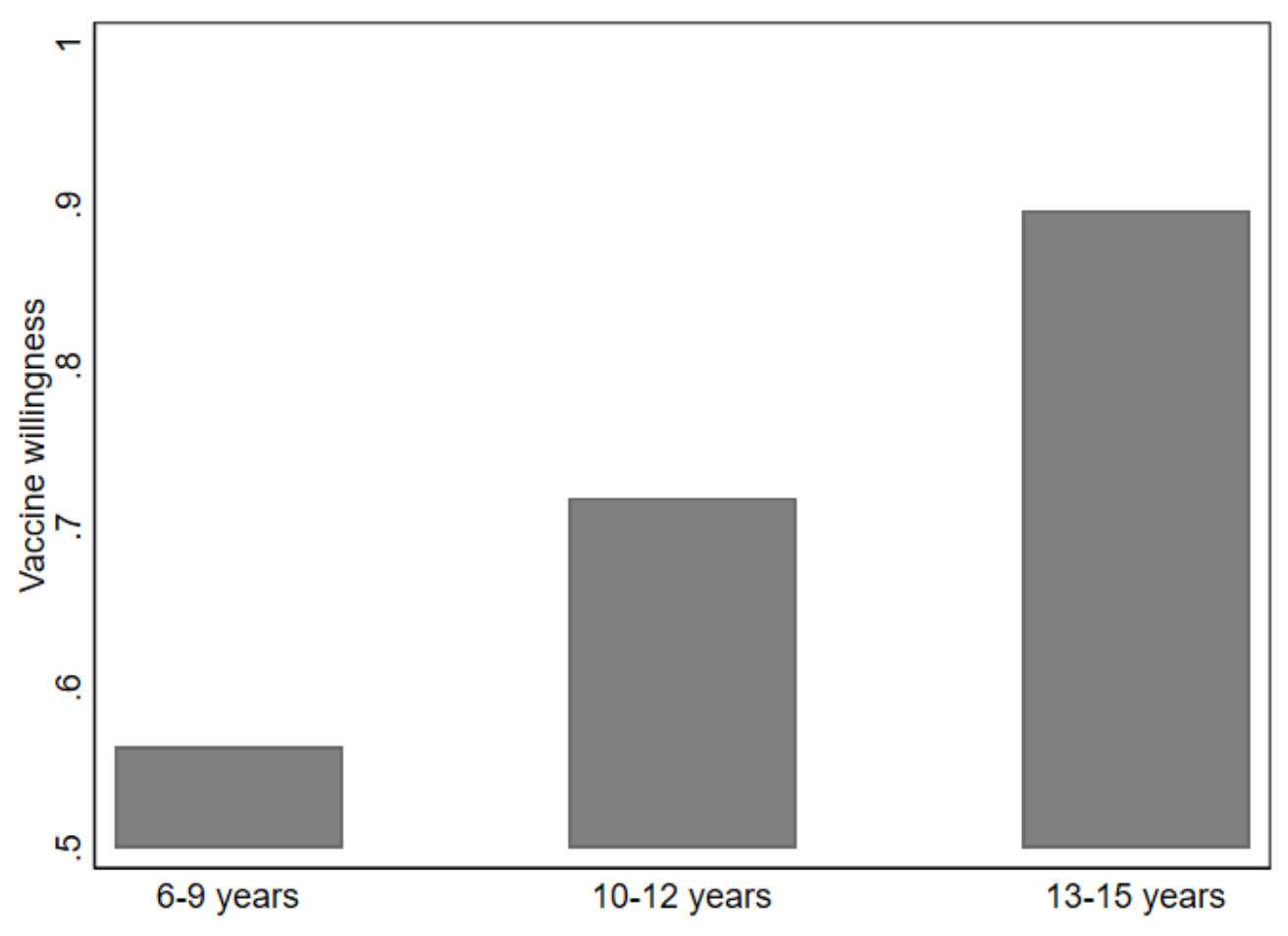

Figure 3 reports the results from our regressions of vaccine willingness on the predictors (Table S.1 reports the supporting regression table, including results from bivariate regressions). Model I, confirms the results from above that there is a positive correlation between vaccine

\footnotetext{
${ }^{1}$ It should be noted that these figures should be considered an absolute upper-bound on vaccine intentions. As of November $272021,73 \%$ of children aged 12-15 have received a first vaccination dose, which is clearly below the estimates provided by the survey. This likely reflects, first, a disjunction between intentions and behavior and, second, the association between vaccine-skepticism and anti-science views (Rutjens et al., 2021), which makes it more difficult to get individuals who are skeptical about vaccines to participate in studies such as the present one.
} 
willingness and the child's age, even controlled for the battery of demographics: the older the child the more likely that parents' accept COVID-19 vaccines for their children. More specifically, controlled for the other demographics parents' are about 13.2 percentage points (95\% CI: 4.3-22.1) more likely to accept vaccines when their child is 10-12 years old compared to 6-9 years old and they are about 30.2 percentage points (95\% CI: 22.4-38.1) more likely to accept vaccines when their child is 12-15 years old. We similarly observe a clear tendency for older parents' to be more willing to vaccinate their children. Focusing on Model I that accounts for the fact that younger parents tend to have younger children, we observe that parents' aged 35-50 are about 16 percentage points (95\% CI: -0.01-32.5) more likely to accept vaccines for their children compared to younger parents' aged 1834. Similarly, parents' aged 51-65 are about 23.4 percentage points (95\% CI: 5.9-40.0) more likely to accept vaccines. We observe quite little heterogeneity with respect to the remaining demographics: in Model I, there are no statistically discernible sex or education differences. Similarly, there is very little geographic heterogeneity across regions: the only difference which is statistically distinguishable from zero is the difference between Nordjylland and the benchmark (the Municipality) with parents' from Nordjylland being about 11.6 percentage points (95\% CI: 2.4-20.8) more likely to accept vaccines. Note that parents' willingness to vaccinate their children in Nordjylland is not statistically different from any of the other regions.

Moving to Model II that includes predispositions, we observe that trust and knowledge are both positively correlated with parents' intention to vaccinate their children against COVID-19. Recall that the correlations reflect a 2 standard deviation increase in trust and knowledge. In particular, a 2 standard deviations increase in trust is associated with about a 20.8 percentage points (95\% CI: 14.3-27.3) increase in vaccine willingness. A 2 standard deviation knowledge increase is associated with about 15.2 percentage points (95\% CI: 8.4-21.9) increase in vaccine willingness.

Figure 3. Vaccine willingness, demographics, predispositions, perceptions, and barriers 


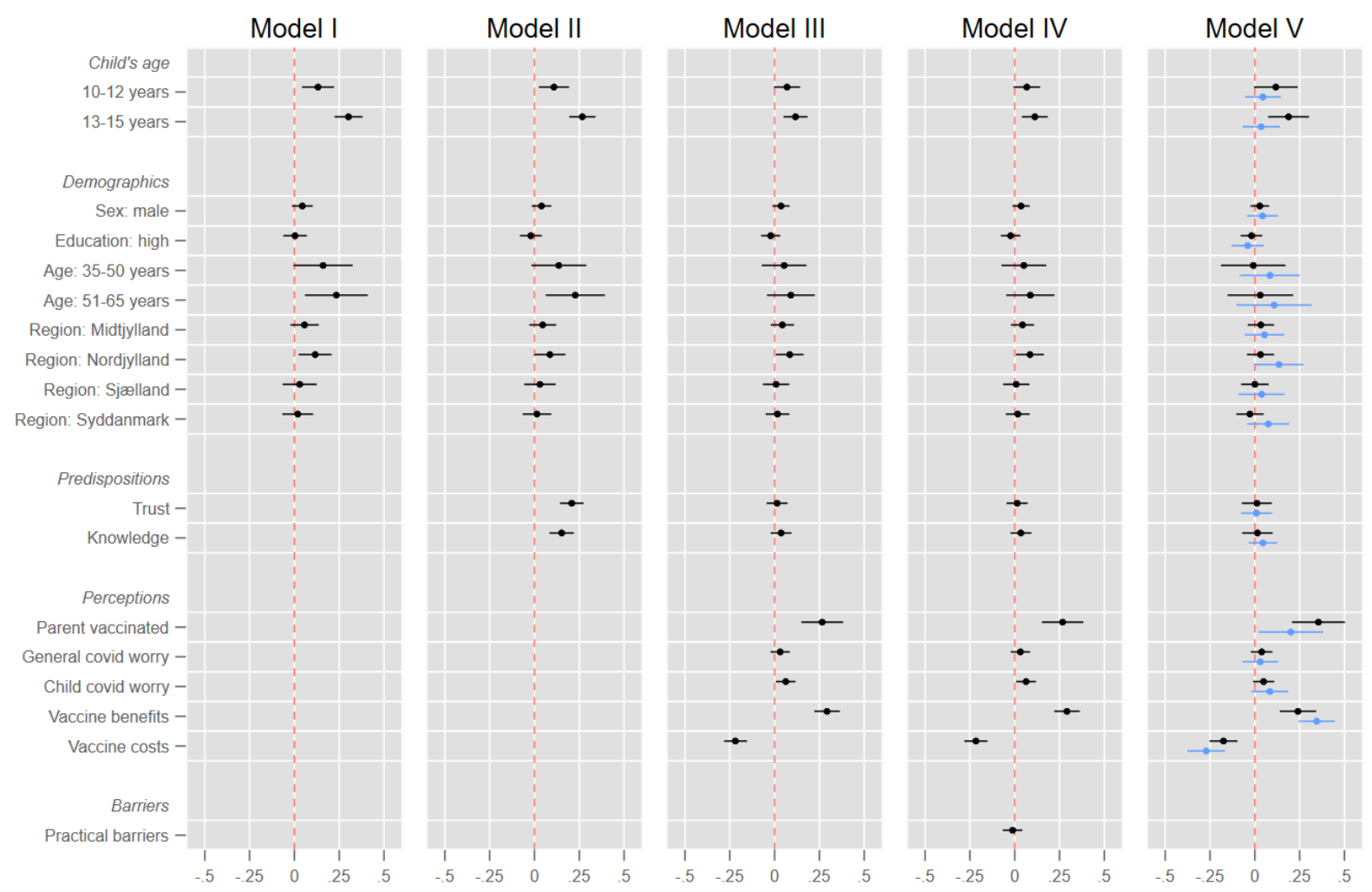

Note: Filled circles are unstandardized regression coefficient with $95 \%$ confidence intervals (robust standard errors). Model V: black filled circles are estimates for parents below the median barrier level while blue filled estimates are for parents above the median level. $\mathrm{N}=794$.

Focusing on Model III that includes perceptions, it is first worth noticing that the inclusion of perceptions seem to fully mediate the estimated associations between predispositions and vaccine willingness. Thus, we observe that the correlations between vaccine willingness and trust and knowledge are statistically indistinguishable from zero in Model III when perceptions are introduced into the model. Similarly, we observe a substantial decrease in the correlation between the child's age and vaccine willingness. Turning attention to the estimated correlations between perceptions and vaccine willingness, we observe that parents' who are themselves vaccinated against coronavirus are about 26.7 percentage points (95\% CI: 15.0-38.2) more likely to also be willing to vaccinate their children--controlled for all the other factors in the model--compared to unvaccinated parents. There is no statistically significant correlation between general COVID worry and vaccine willingness. In contrast, a 2 standard deviation increase in parents' appraisal of child COVID worry is associated with statistically significant increase in vaccine willingness of about 6.2 percentage points (95\% CI: 0.8-11.7). A 2 standard deviation increase in the perceived benefits of vaccination for the child is 
associated with a whopping 29.3 percentage points (95\% CI: 22.2-36.4) increase in willingness while an increase in the perceived cost of vaccination is associated with a decrease in willingness of about 21.9 percentage points (95\% CI: 15.5-28.2).

Incorporating barriers into the estimations in Model IV as an independent predictor of vaccine willingness does very little to the remaining correlations. Simultaneously, the estimated correlation between barriers and vaccine willingness itself is very close to zero and far from being statistically significant.

In Model V, we re-estimate Model III while splitting the sample into parents' above (blue filled circles) and below (black filled circles) the median on perceived barriers for vaccination in order to test the proposition that barriers moderate the relationship between perceptions and vaccine willingness. Here we do observe some heterogeneity with respect to especially parent vaccination, vaccine benefits, and vaccine costs. In particular, the positive correlation between parent vaccine and vaccine willingness seems to be driven mainly by parents below the median: among parents below the median there is about a 35.5 percentage points (95\% CI: 20.7-50.2) difference in vaccine willingness between the vaccinated and unvaccinated while the difference is about 20.1 percentage points (95 \% CI: 2.1-38.1) among parent above the median. On vaccine benefits, the negative correlation seems to be driven by those above the barrier median: among parents above the median, a 2 standard deviation increase in benefits is associated with a 34.5 percentage points increase in willingness (95\% CI: 24.4-44.6) while the corresponding increase is 24.1 percentage points (95\% CI: 14.0-34.2) among parents below the median. We observe the same pattern on vaccine cost: an increase in costs is associated with a decrease of about 27.1 percentage point (95\% CI: 16.6-34.5) among parents above the median and a 17.5 percentage points (95\% CI: 9.7-25.2) decrease among parents below the median. Note, however, that when formally modelling these differential correlations the interactions are not statistically significantly different from zero at the 5 percent confidence level.

\section{Discussion and Conclusion}

These results demonstrate that parents' intentions to get their children vaccinated against COVID-19, in the end, boils down to a calculation of the costs and benefits of the vaccination for the children. The costs involved a range of perceptions related to side-effects of COVID-19 vaccines and, given these perceptions, a perception that natural immunity may be superior to vaccine-induced immunity. The benefits involve perceptions relating to both the societal benefits in the form of reaching herd 
immunity and protection of others and the personal benefits for the child in the form of normalizing life for the child. Beyond the variance explained by these factors, the parent's own decision to become vaccinated also has a substantial and independent impact on vaccination intentions with regards to their children.

The stepwise logic of the analysis suggests that perceptions of the costs and benefits of the vaccines, in part, reflect more foundational background characteristics. In particular, parents are more likely to be hesitant (likely because of greater concerns regarding the costs of vaccines) when the child is younger. Furthermore, general knowledge about COVID-19 vaccines and general trust in the health authorities constitutes background factors that predispose parents towards vaccinations, most likely by affecting both their own vaccination status and their perceptions of the costs and benefits of the vaccines. It is relevant to note how the stepwise logic of the analysis was key to demonstrate these findings. If all factors had been entered concomitantly (as in Figure 3, Model IV), one would have been inclined to accept the (false) conclusion that trust and knowledge was not associated with vaccination intentions.

Just as it is important to understand the factors that matter for parental decisions regarding the vaccination of children against COVID-19, it is important to understand the factors that matter less. In this regard, it seems that perceptions of the disease itself have much lower explanatory power than perceptions of the disease. Furthermore, practical barriers have little moderating effects on the relationship between perceptions and intentions.

Overall, these findings suggest that, even in a country with high vaccine-uptake such as Denmark, there will be substantial hesitancy towards vaccinating young children against COVID-19. This implies that we may expect even greater hesitancy in countries that are facing greater struggles with skepticism against vaccines. Furthermore, to increase the uptake of vaccines that have been validated as safe and efficient, the findings suggest that health authorities should focus less on communication regarding the disease of COVID-19 itself and more on communication regarding the vaccines. First, it is of key importance to make sure that parental concerns about side-effects are acknowledged and addressed. Second, it is of key importance that parents have an adequate understanding of how vaccination of their children will help normalize both the functions of society in general and their children's' everyday lives. 


\section{References}

Bartsch, S. M., O'Shea, K. J., Ferguson, M. C., Bottazzi, M. E., Wedlock, P. T., Strych, U., ... \& Lee, B. Y. (2020). Vaccine efficacy needed for a COVID-19 coronavirus vaccine to prevent or stop an epidemic as the sole intervention. American journal of preventive medicine, 59(4), 493-503.

Bell, S., Clarke, R., Mounier-Jack, S., Walker, J. L., \& Paterson, P. (2020). Parents' and guardians' views on the acceptability of a future COVID-19 vaccine: A multi-methods study in England. Vaccine, 38(49), 7789-7798.

Brandstetter, S., Böhmer, M. M., Pawellek, M., Seelbach-Göbel, B., Melter, M., Kabesch, M., \& Apfelbacher, C. (2021). Parents' intention to get vaccinated and to have their child vaccinated against COVID-19: cross-sectional analyses using data from the KUNO-Kids health study. European journal of pediatrics, 1-6.

Britton, T., Ball, F., \& Trapman, P. (2020). A mathematical model reveals the influence of population heterogeneity on herd immunity to SARS-CoV-2. Science, 369(6505), 846-849.

Campbell, A., Converse, P. E., Miller, W. E., \& Stokes, D. E. (1980). The american voter. University of Chicago Press.

Galanis, P., Vraka, I., Siskou, O., Konstantakopoulou, O., Katsiroumpa, A., \& Kaitelidou, D. (2021). Willingness and influential factors of parents to vaccinate their children against the COVID19: a systematic review and meta-analysis. medRxiv.

Goldman, R. D., Yan, T. D., Seiler, M., Cotanda, C. P., Brown, J. C., Klein, E. J. \& Staubli, G. (2020). Caregiver willingness to vaccinate their children against COVID-19: Cross sectional survey. Vaccine, 38(48), 7668-7673.

Hetherington, E., Edwards, S. A., MacDonald, S. E., Racine, N., Madigan, S., McDonald, S., \& Tough, S. (2021). SARS-CoV-2 vaccination intentions among mothers of children aged 9 to 12 years: a survey of the All Our Families cohort. CMAJ open, 9(2), E548.

Jørgensen, F. J., Bor, A., \& Petersen, M. B. (2021). How the Development, Features and Roll-Out of a SARS-COV-2 Vaccine Shape Public Acceptance: A Conjoint Experiment in a Large Representative Sample of Danes.

Kaplan, R. M., \& Milstein, A. (2021). Influence of a COVID-19 vaccine's effectiveness and safety profile on vaccination acceptance. Proceedings of the National Academy of Sciences, 118(10). 
Kelly, B. J., Southwell, B. G., McCormack, L. A., Bann, C. M., MacDonald, P. D., Frasier, A. M., ... \& Squiers, L. B. (2021). Predictors of willingness to get a COVID-19 vaccine in the US. BMC Infectious Diseases, 21(1), 1-7.

Kreps, S., Prasad, S., Brownstein, J. S., Hswen, Y., Garibaldi, B. T., Zhang, B., \& Kriner, D. L. (2020). Factors associated with US adults' likelihood of accepting COVID-19 vaccination. JAMA network open, 3(10), e2025594-e2025594.

Montalti, M., Rallo, F., Guaraldi, F., Bartoli, L., Po, G., Stillo, M., .. \& Gori, D. (2021). Would Parents Get Their Children Vaccinated Against SARS-CoV-2? Rate and Predictors of Vaccine Hesitancy According to a Survey over 5000 Families from Bologna, Italy. Vaccines, 9(4), 366.

Motta, M. (2021). Can a COVID-19 vaccine live up to Americans' expectations? A conjoint analysis of how vaccine characteristics influence vaccination intentions. Social Science \& Medicine, 113642.

Ruggiero, K. M., Wong, J., Sweeney, C. F., Avola, A., Auger, A., Macaluso, M., \& Reidy, P. (2021). Parents' Intentions to Vaccinate Their Children Against COVID-19. Journal of Pediatric Health Care, 35(5), 509-517.

Rutjens, B. T., van der Linden, S., \& van der Lee, R. (2021). Science skepticism in times of COVID-19. Group Processes \& Intergroup Relations, 24(2), 276-283.

Sanche, S., Lin, Y. T., Xu, C., Romero-Severson, E., Hengartner, N., \& Ke, R. (2020). High contagiousness and rapid spread of severe acute respiratory syndrome coronavirus 2. Emerging infectious diseases, 26(7), 1470.

Scherer, A. M., Gedlinske, A. M., Parker, A. M., Gidengil, C. A., Askelson, N. M., Petersen, C. A., ... \& Lindley, M. C. (2021). Acceptability of adolescent COVID-19 vaccination among adolescents and parents of adolescents-United States, April 15-23, 2021. Morbidity and Mortality Weekly Report, 70(28), 997.

Schwarzinger, M., Watson, V., Arwidson, P., Alla, F., \& Luchini, S. (2021). COVID-19 vaccine hesitancy in a representative working-age population in France: a survey experiment based on vaccine characteristics. The Lancet Public Health.

Skjefte, M., Ngirbabul, M., Akeju, O., Escudero, D., Hernandez-Diaz, S., Wyszynski, D. F., \& Wu, J. W. (2021). COVID-19 vaccine acceptance among pregnant women and mothers of young children: results of a survey in 16 countries. European journal of epidemiology, 36(2), 197-211. 
Szilagyi, P. G., Shah, M. D., Delgado, J. R., Thomas, K., Vizueta, N., Cui, Y., ... \& Kapteyn, A. (2021). Parents' intentions and perceptions about COVID-19 vaccination for their children: results from a national survey. Pediatrics, 148(4).

Tanne, J. H. (2021). Covid-19: Cases in children rise sharply in US as doctors call for vaccine approval. BMJ: British Medical Journal (Online), 374.

Teasdale, C. A., Borrell, L. N., Kimball, S., Rinke, M. L., Rane, M., Fleary, S. A., \& Nash, D. (2021). Plans to Vaccinate Children for COVID-19: A Survey of United States Parents. The Jpurnal of Pediatrics.

Xu, Y., Zhang, R., Zhou, Z., Fan, J., Liang, J., Cai, L., ... \& Lin, W. (2021). Parental psychological distress and attitudes towards COVID-19 vaccination: a cross-sectional survey in Shenzhen, China. Journal of Affective Disorders.

Yigit, M., Ozkaya-Parlakay, A., \& Senel, E. (2021). Evaluation of COVID-19 vaccine refusal in parents. The Pediatric Infectious Disease Journal, 40(4), e134-e136.

Y1lmaz, M., \& Sahin, M. K. (2021). Parents' willingness and attitudes concerning the COVID-19 vaccine: A cross-sectional study. International Journal of Clinical Practice, e14364.

Zaller, J. R. (1992). The nature and origins of mass opinion. Cambridge University Press.

Zhang, K. C., Fang, Y., Cao, H., Chen, H., Hu, T., Chen, Y. Q., ... \& Wang, Z. (2020). Parental acceptability of COVID-19 vaccination for children under the age of 18 years: cross-sectional online survey. JMIR pediatrics and parenting, 3(2), e24827.

WHO (2021). Statement - WHO/Europe: stagnating COVID-19 vaccination uptake requires urgent action. https://www.euro.who.int/en/media-centre/sections/statements/2021/statement-whoeuropestagnating-covid-19-vaccination-uptake-requires-urgent-action, retrieved November 132021. 
Supplementary Information

Table S.1. Supporting regression table

\begin{tabular}{|c|c|c|c|c|c|c|c|}
\hline & $\begin{array}{c}\text { Bivariate } \\
\text { correlations }\end{array}$ & Model 1 & Model 2 & Model 3 & Model 4 & Model 5a & Model 5b \\
\hline $\begin{array}{l}\text { Child age: } \\
6-9\end{array}$ & ref & ref & ref & ref & ref & ref & ref \\
\hline Child age: & $0.16^{* * *}$ & $0.13^{* *}$ & $0.11^{*}$ & 0.07 & 0.07 & 0.12 & 0.04 \\
\hline $10-12$ & $(0.04)$ & $(0.05)$ & $(0.04)$ & $(0.04)$ & $(0.04)$ & $(0.06)$ & $(0.05)$ \\
\hline Child age: & $0.33^{* * * *}$ & $0.30^{* * * *}$ & $0.27^{* * * *}$ & $0.12^{* * * *}$ & $0.11^{* *}$ & $0.19^{* *}$ & 0.04 \\
\hline $13-15$ & $(0.04)$ & $(0.04)$ & $(0.04)$ & $(0.03)$ & $(0.04)$ & $(0.06)$ & $(0.05)$ \\
\hline Sex: female & ref & ref & ref & ref & ref & ref & ref \\
\hline \multirow[t]{2}{*}{ Sex: male } & 0.05 & 0.04 & 0.04 & 0.04 & 0.04 & 0.03 & 0.04 \\
\hline & $(0.03)$ & $(0.03)$ & $(0.03)$ & $(0.02)$ & $(0.02)$ & $(0.03)$ & $(0.04)$ \\
\hline $\begin{array}{l}\text { Education: } \\
\text { low }\end{array}$ & ref & ref & ref & ref & ref & ref & ref \\
\hline Education: & -0.02 & 0.00 & -0.02 & -0.02 & -0.02 & -0.02 & -0.04 \\
\hline high & $(0.04)$ & $(0.03)$ & $(0.03)$ & $(0.03)$ & $(0.03)$ & $(0.03)$ & $(0.05)$ \\
\hline Age: $18-34$ & Ref & ref & ref & ref & ref & ref & ref \\
\hline \multirow[t]{2}{*}{ Age: $35-50$} & $0.25^{* * *}$ & 0.16 & 0.14 & 0.05 & 0.05 & -0.01 & 0.08 \\
\hline & $(0.08)$ & (0.08) & $(0.08)$ & $(0.06)$ & $(0.06)$ & (0.09) & $(0.08)$ \\
\hline \multirow[t]{2}{*}{ Age: 51-65 } & $0.40^{* * *}$ & $0.23^{* *}$ & $0.23^{* *}$ & 0.09 & 0.09 & 0.03 & 0.11 \\
\hline & $(0.08)$ & (0.09) & $(0.08)$ & $(0.07)$ & $(0.07)$ & (0.09) & $(0.11)$ \\
\hline \multicolumn{8}{|l|}{ Municipality } \\
\hline Region: & 0.08 & 0.06 & 0.05 & 0.04 & 0.04 & 0.03 & 0.05 \\
\hline Midtjylland & $(0.04)$ & $(0.04)$ & $(0.04)$ & $(0.03)$ & $(0.03)$ & $(0.04)$ & $(0.06)$ \\
\hline Region: & $0.13^{*}$ & $0.12^{*}$ & 0.09 & $0.08^{*}$ & $0.09^{*}$ & 0.03 & 0.13 \\
\hline Nordjylland & $(0.05)$ & $(0.05)$ & $(0.04)$ & $(0.04)$ & $(0.04)$ & $(0.04)$ & $(0.07)$ \\
\hline Region: & 0.05 & 0.03 & 0.03 & 0.01 & 0.01 & 0.00 & 0.04 \\
\hline Sjælland & $(0.05)$ & $(0.05)$ & $(0.04)$ & $(0.04)$ & $(0.04)$ & $(0.04)$ & (0.07) \\
\hline Region: & 0.04 & 0.02 & 0.01 & 0.02 & 0.02 & -0.03 & 0.07 \\
\hline Syddanmark & $(0.03)$ & $(0.04)$ & $(0.04)$ & $(0.03)$ & $(0.03)$ & $(0.04)$ & $(0.06)$ \\
\hline \multirow[t]{2}{*}{ Trust } & $0.28^{* * *}$ & & $0.21^{* * *}$ & 0.01 & 0.01 & 0.01 & 0.01 \\
\hline & $(0.03)$ & & $(0.03)$ & $(0.03)$ & $(0.03)$ & $(0.04)$ & $(0.04)$ \\
\hline \multirow[t]{2}{*}{ Knowledge } & $0.23^{* * *}$ & & $0.15^{* * *}$ & 0.04 & 0.03 & 0.01 & 0.05 \\
\hline & $(0.03)$ & & $(0.03)$ & $(0.03)$ & $(0.03)$ & $(0.04)$ & $(0.04)$ \\
\hline Parent & $0.68^{* * *}$ & & & $0.27^{* * * *}$ & $0.27^{* * *}$ & $0.35^{* * *}$ & $0.20^{*}$ \\
\hline vaccinated & $(0.05)$ & & & $(0.06)$ & $(0.06)$ & $(0.08)$ & (0.09) \\
\hline General & $0.14^{* * *}$ & & & 0.03 & 0.03 & 0.04 & 0.03 \\
\hline covid worry & $(0.03)$ & & & $(0.03)$ & $(0.03)$ & $(0.03)$ & $(0.05)$ \\
\hline Child covid & $0.17^{* * *}$ & & & $0.06^{*}$ & $0.06^{*}$ & 0.05 & 0.08 \\
\hline worry & $(0.03)$ & & & $(0.03)$ & $(0.03)$ & $(0.03)$ & $(0.05)$ \\
\hline Vaccine & $0.50^{* * *}$ & & & $0.29^{* * * *}$ & $0.29^{* * *}$ & $0.24^{* * *}$ & $0.35^{* * *}$ \\
\hline benefits & $(0.04)$ & & & $(0.04)$ & $(0.04)$ & $(0.05)$ & $(0.05)$ \\
\hline Vaccine & $-0.45^{* * *}$ & & & $-0.22^{* * * *}$ & $-0.22^{* * *}$ & $-0.17^{* * *}$ & $-0.27^{* * *}$ \\
\hline costs & $(0.03)$ & & & $(0.03)$ & $(0.03)$ & $(0.04)$ & $(0.05)$ \\
\hline Vaccine & $-0.24^{* * *}$ & & & & -0.01 & & \\
\hline barriers & $(0.03)$ & & & & $(0.03)$ & & \\
\hline \multirow[t]{2}{*}{ Constant } & & $0.32^{* *}$ & $0.41^{* * *}$ & $0.33^{* *}$ & $0.34^{* *}$ & $0.29^{*}$ & $0.40^{*}$ \\
\hline & & $(0.11)$ & $(0.11)$ & $(0.10)$ & $(0.10)$ & $(0.12)$ & $(0.16)$ \\
\hline$N$ & 794 & 794 & 794 & 794 & 794 & 427 & 367 \\
\hline $\operatorname{adj} . R^{2}$ & & 0.108 & 0.219 & 0.434 & 0.434 & 0.473 & 0.359 \\
\hline
\end{tabular}


Figures S.1. Replicating the main results using logistic regression
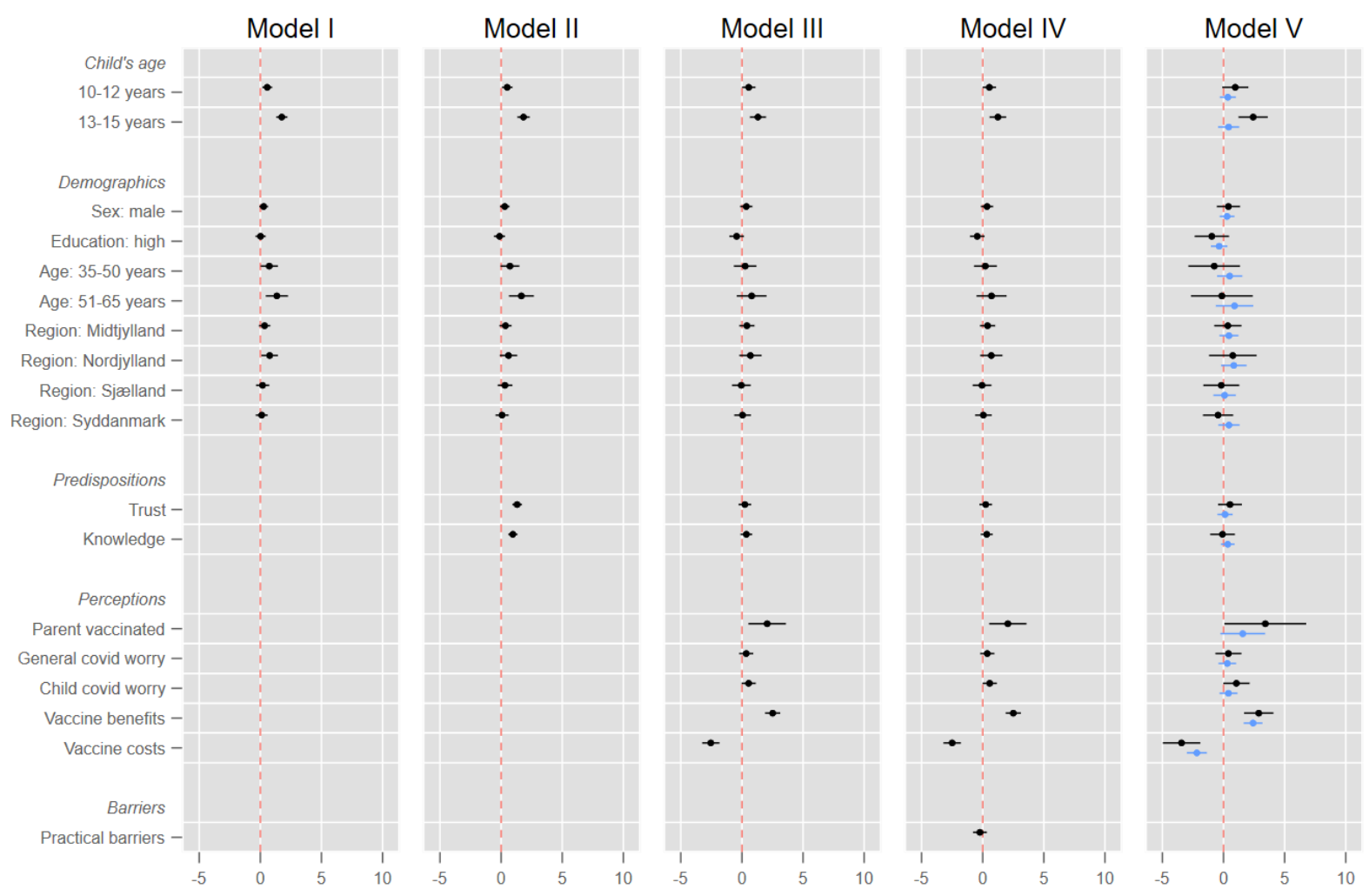

Note: Filled circles are logistic regression coefficients with $95 \%$ confidence intervals. Model V: black filled circles are estimates for parents below the median barrier level while blue filled estimates are for parents above the median level. $\mathrm{N}$ $=794$. 
Figures S.2. Replicating main results while excluding “don't know" responses

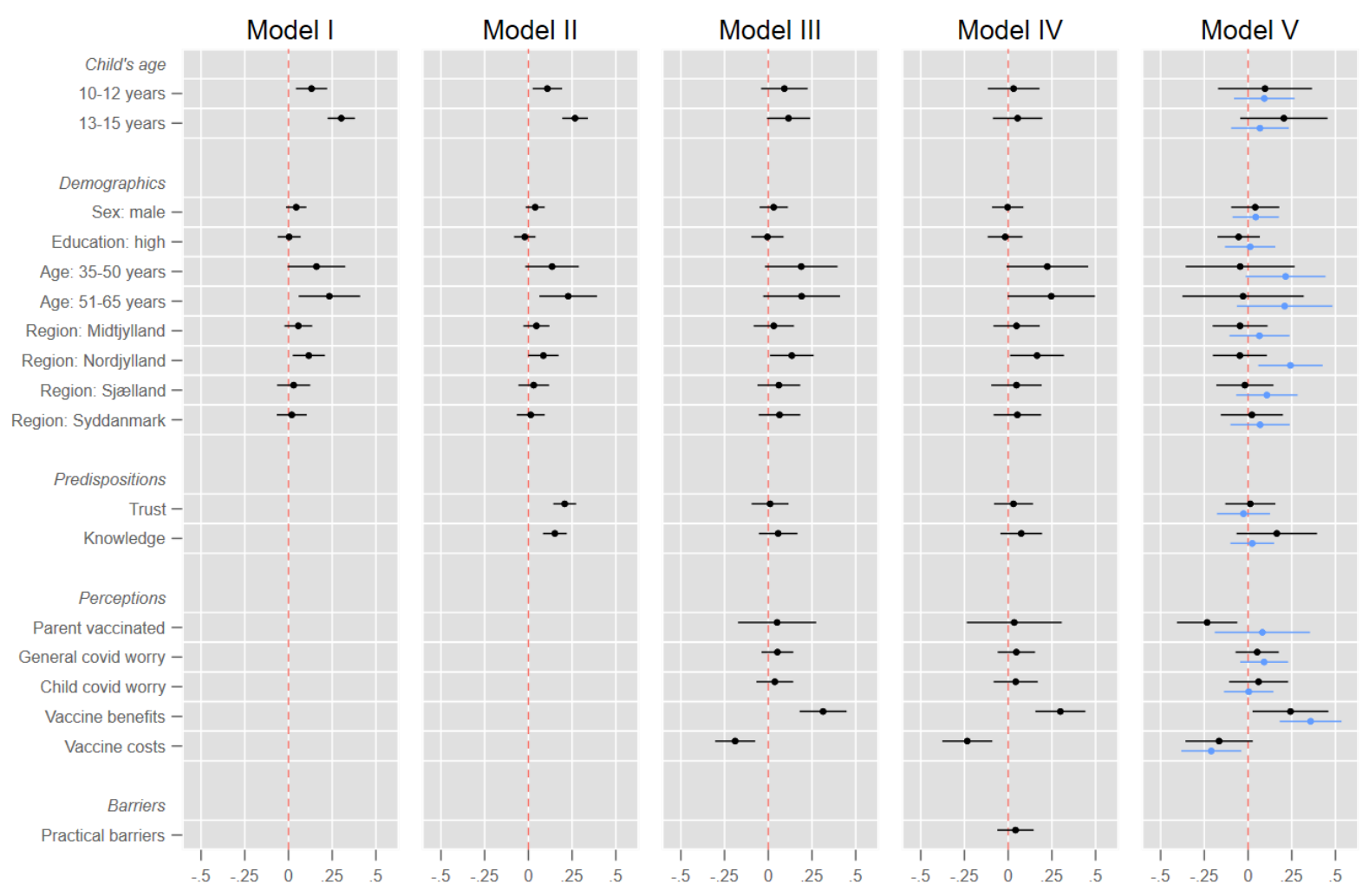

Note: Filled circles are unstandardized regression coefficient with $95 \%$ confidence intervals (robust standard errors). Model V: black filled circles are estimates for parents below the median barrier level while blue filled estimates are for parents above the median level. 